University of Louisville

ThinkIR: The University of Louisville's Institutional Repository

Faculty Scholarship

$11-1-2019$

\title{
Default parallels: The science potential of JWST parallel observations during TSO primary observations
}

\author{
Benne W. Holwerda \\ University of Louisville, benne.holwerda@louisville.edu \\ Jonathan Fraine \\ STSCl \\ Nelly Mouawad \\ Lebanese American University \\ Joanna S. Bridge \\ University of Louisville
}

Follow this and additional works at: https://ir.library.louisville.edu/faculty

Part of the Astrophysics and Astronomy Commons

\section{ThinkIR Citation}

Holwerda, Benne W.; Fraine, Jonathan; Mouawad, Nelly; and Bridge, Joanna S., "Default parallels: The science potential of JWST parallel observations during TSO primary observations" (2019). Faculty Scholarship. 481.

https://ir.library.louisville.edu/faculty/481

This Article is brought to you for free and open access by ThinkIR: The University of Louisville's Institutional Repository. It has been accepted for inclusion in Faculty Scholarship by an authorized administrator of ThinkIR: The University of Louisville's Institutional Repository. For more information, please contact thinkir@louisville.edu. 


\title{
Default Parallels: The Science Potential of JWST Parallel Observations during TSO Primary Observations
}

\author{
B. W. Holwerda ${ }^{1}$ (D) , Jonathan Fraine ${ }^{2,3}$, Nelly Mouawad ${ }^{4}$ (D), and Joanna S. Bridge ${ }^{1}$ (D) \\ ${ }^{1}$ Department of Physics and Astronomy, University of Louisville, 102 Natural Science Building, Louisville, KY 40292, USA; benne.holwerda@louisville.edu \\ ${ }^{2}$ Space Telescope Science Institute, 3700 San Martin Drive, Baltimore, MD 21218, USA \\ ${ }^{3}$ Space Science Institute, 4750 Walnut Street, Suite 205, Boulder, CO 80301, USA \\ ${ }^{4}$ Lebanese American University, 211 East 46th Street, New York, NY 10017, USA \\ Received 2018 November 28; accepted 2019 July 17; published 2019 October 15
}

\begin{abstract}
The James Webb Space Telescope (JWST) will observe several stars for long cumulative durations while pursuing exoplanets as primary science targets for both Guaranteed Time Observations (GTO) and very likely General Observer (GO) programs. Here we argue in favor of an automatic default parallel program to observe, e.g., using the F200W/F277W filters or grism of NIRCAM/NIRISS in order to find high redshift $(z \gg 10)$ galaxies, cool red/brown dwarf substellar objects, solar system objects, and observations of serendipitous planetary transits. We argue here the need for automated exploratory astrophysical observations with unused JWST instruments during these long-duration exoplanet observations. Randomized fields that are observed in parallel mode reduce errors due to cosmic variance more effectively than single continuous fields of a typical wedding cake observing strategy. Hence, we argue that the proposed automated survey will explore a unique and rich discovery space in the highredshift universe, Galactic structure, and solar system. We show that the GTO and highly probable GO target list of exoplanets covers the Galactic disk/halo and high redshift universe, mostly well out of the plane of the disk of the Milky Way. Exposure times are of the order of the CEERS GTO medium-deep survey in a single filter, comparable to CANDELS in Hubble Space Telescope's surveys and deep fields. The area covered by NIRISS and NIRCam combined could accumulate to a half square degree surveyed.
\end{abstract}

Key words: galaxies: active - galaxies: evolution - galaxies: high-redshift - Galaxy: stellar content - methods: statistical - space vehicles: instruments - surveys - techniques: imaging spectroscopy

Online material: color figures

\section{Introduction}

Given the high price tag and the limited life expectancy of the James Webb Space Telescope (JWST), the pressure is on the astronomical community to maximize this new flagship's efficiency. Two observational communities that will make maximum the use of this observatory and its revolutionary instrument suite are the exoplanet and the high-redshift galaxy population communities. Exoplanet direct imaging and transit studies require long, single-pointing exposures. Meanwhile, the other instruments on JWST will be left unused for science. Given reasonable use (i.e., no filter changes, long integrations to limit buffer use), these can be put to excellent use for solar system, Galactic, and extragalactic science (Figure 1).

In order to minimize resources allocated to this program, we propose a single strategy to simplify the generation of this legacy data set, "default parallels."

Default parallels would be planned as follows:

1. Automatically examine in each nonprimary instrument's field of view (FOV).
2. If there is a bright target that would saturate deep photometry, then perform a transit observation (i.e., a time series of imaging with repeated short integrations).

3. If there are no bright sources, then do a deep (extragalactic) observation in a band redder than $H$, or using grism observations.

4. If the number of intermezzos is above three or four, deep imaging with multiple filters is performed.

5. If not enough principal observation intermezzos are available for deep imaging, grism observations are performed.

The possibility of default parallels also would have to be calculated with the boundary conditions of no or few filter changes, no dithering, long integrations, and within JWST's limited onboard data storage space. Given the existing exoplanet JWST Guaranteed Time Observations (GTO), ERS, and prospective General Observer (GO) observational expectations, it is possible that a default parallel program could observe up to a half a square degree - accumulated area-for deep galactic fields $(m<28)$ in either the F277W or F200W 


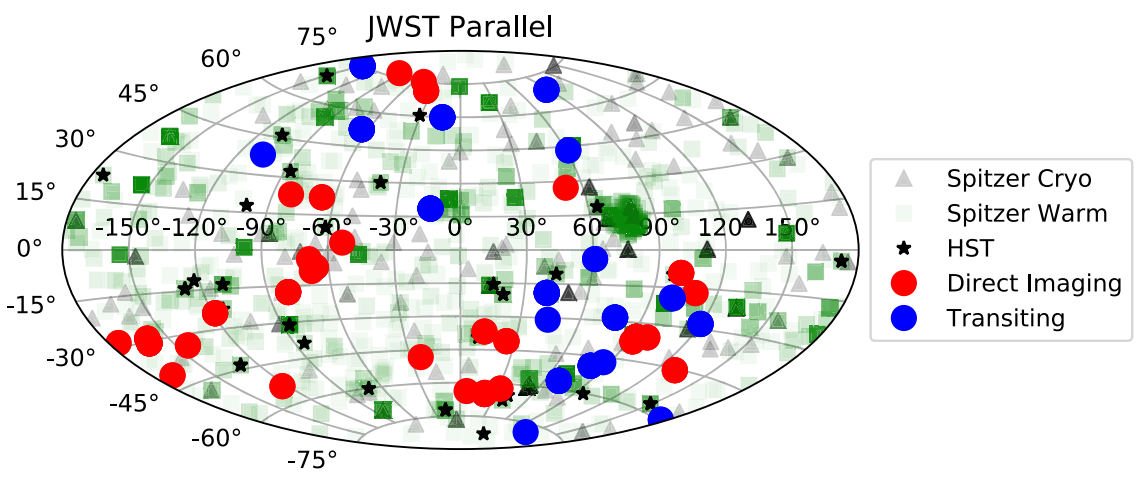

Figure 1. Spitzer Cryo and Warm mission transient targets and HST GO exoplanet programs (likely targets for JWST GO follow-up proposals) and JWST exoplanet GTO programs projected on the sky in galactic coordinates. While there are multiple programs on a variety of targets, the number of pointings is $\sim 30$ for the GTO (blue and red dots) and unknown for the GO programs (depending on the GO success rate).

(A color version of this figure is available in the online journal.)

filter from NIRISS and NIRCam, spanning a large range of sky coverage; these filters were selected because they are available on both instruments. We highlight double NIRISS and NIRCam observations as these can be homogeneous and wide field $\left(2 \times\left(3^{\prime} \times 3^{\prime}\right) \mathrm{FOV}\right)$ with limiting magnitudes ranging from $m_{A B} \sim 27$ to $29 \mathrm{mag}$ (depending on actual in-flight performance, etc.).

Mid-InfraRed Instrument (MIRI) observations (FOV 74" $\times$ $113^{\prime \prime}$ ) in default parallels would provide separate unique, long wavelength coverage not available with any NIR instruments. Below are a few science cases for such observations.

\section{Default Parallel Science Cases}

\subsection{Science Case A: High-redshift Galaxies}

Long-stare NIRcam or NIRISS F200W/F277W observations would be beyond the $H$-band of Euclid or the F184 filter on WFIRST, or Hubble's F160W filter. These images will effectively search for $H$-band dropout galaxies $(z>11)$. Without ancillary data, these observations would be of limited immediate use for high-redshift studies, but with solid $H$-band fluxes, this could be an excellent constraint on the luminosity function of $z>11$ galaxy populations using the Lyman break technique (Steidel et al. 1996). Critical here is that the $H$-band observations can be a follow-up of these JWST parallel fields. The value of these preliminary search observations is a key investment for the discovery space of future follow-up observations.

A similar program of Hubble Space Telescope (HST)/WFC3 default parallels has been extremely successful in constraining the $z \sim 8$ and $z \sim 9$ brighter populations (Trenti et al. 2011, 2012; Bradley et al. 2012; Trenti 2014; Bernard et al. 2016; Calvi et al. 2016; Livermore et al. 2018; Morishita et al. 2018), taking statistical power from the essentially random pointings, and therefore sampling a large volume (Trenti \& Stiavelli 2008).
The random sampling also resulted in useful lower-redshift $(z \sim 2)$ clustering constraints (Cameron et al. 2019), which would have been difficult to obtain otherwise. Similar searches can be done with just JWST F200W/F277W observations using NIRISS/NIRCam, supplemented with WFIRST/EUCLID/HST near-infrared $(J$ - and $H$-band) follow-up.

During exoplanet phase-curve observations and high-contrast imaging programs, there are nominally re-pointings to change filters (direct imaging) or data downloads (phase curves). During these significantly longer exposures, with sufficiently long intermezzos for re-pointings or data downloads, multiple filter observations can be considered for the nonprimary instrument's default parallels, for example, during transiting observations that consist of the greatest time commitments. We assume that during sensitive exoplanet observations, a filter change on another instrument would be too disruptive and these should be reserved for breaks in the primary observation. If no break is available, the parallel would be a single F200W or F277W deep image, with the longest on-ramp integrations for minimal storage use. Multiple filter parallel observations (i.e., with primary observation breaks for filter changes in the parallel) would expand the search space to $z \gg 11$ sources. These would be of almost similar quality as dedicated deep fields, without the specifically optimized dithering strategy, however.

\subsection{Science Case B: Brown Dwarf Population of the Milky Way}

Similarly, a search for the lowest-mass substellar objects belonging to the Milky Way can benefit from observations such as the above default parallels. The randomized sampling of the Milky Way volume equally benefits constraints on the shape of the Milky Way (Pirzkal et al. 2005, 2009; Ryan et al. 2005, 2011, 2017; Holwerda et al. 2014, 2018; van Vledder et al. 2016). The near-infrared observations of JWST are sensitive to 
the lowest-mass objects (Y dwarfs) throughout the width of the Galactic disk and halo.

The randomized nature of the fields would allow for a first survey of Galactic disk and halo substellar objects, down to freefloating super-Jupiters (e.g., Ryan et al. 2017; Deacon 2018). These parallel searches for low/substellar objects benefit from color information (Holwerda et al. 2018), accurate astrometry, or grism information, but they derive their statistical power from the randomized fields.

\subsection{Science Case C: Solar System Objects}

The existence of default parallels increases the chance to discover or follow-up on solar system objects during deep stares. Meaningful, accidental science occurred during deep field observations with Spitzer, for example, when Spitzer/ MIPS observations caught a passing asteroid (Meadows et al. 2004; Ryan et al. 2015). The likelihood of near-infrared telescopes catching solar system objects is decidedly nonzero (Kiss et al. 2008). Spitzer has performed several successful targeted observations of solar system objects (Stansberry et al. 2004; Fernández et al. 2013; Kelley et al. 2013; Trilling et al. $2016,2017)$ as well as a study of solar system bodies in the ecliptic (Meadows et al. 2004). As such, serendipitous and targeted programs on JWST are fully complementary.

Kuiper Belt objects (KBOs) can be identified in default parallels. High-inclination KBOs, out of the ecliptic plane, are especially of interest (e.g., Batygin \& Brown 2016). This would include objects similar to the $\mathrm{KBO}$ that was just visited by the New Horizons spacecraft, which has an $R$-band magnitude around 26-27, similar to the limiting magnitude for the default parallels program (Figure 5). Moreover, Petit et al. (2008) predict that $\sim 1 \mathrm{KBO}$ object should exist per $\mathrm{deg}^{2}$ with an $R$-band magnitude brighter than 23-23.5. Petit et al. (2011) further estimated an observable population of KBOs with $m_{g} \sim 23-24.5$ per $\mathrm{deg}^{2}$. By covering a large number of deep, randomized fields, the default parallels program would have a significant possibility of discovering and quantifying the distribution of solar system objects.

$\mathrm{KBO}$ require at least two epochs for identification, and we point out that many of the exoplanet observations are scheduled in multiple epochs as well. There will have to be some serendipity here (parallel on the same pointing and position angle). However, if the JWST default parallels represent a single epoch (e.g., F200W observations) the follow-up with EUCLID/WFIRST or HST could represent both the necessary secondary epoch as well as the $H$-band observation for the $H$-band dropout of high redshift galaxies.

\subsubsection{Small Body Discovery and Characterization}

As has been shown by the Kepler, K2, and Transiting Exoplanet Survey Satellite (TESS) surveys-as well as the prediction from JWST yield estimates-almost every FOV near the ecliptic is likely to be dominated by "asteroid trails" from main belt and outer solar system small bodies (Wright et al. 2010). Default parallels would provide a fourfold benefit to the planetary and astronomical communities:

1. During deep imaging, the moving object trails would be used for discovery space. There are likely to be hundreds of thousands of faint solar system objects that have not yet been discovered, especially those emitting at the $J W S T$-MIRI wavelengths $(15-24 \mu \mathrm{m})$.

2. Grism (WFSS) observations with the nonprimary instruments to spectroscopically characterize moving objects in the solar system: KBOs, asteroids, and comets.

3. The randomized FOV will provide direct capture of the distribution and population of small bodies in our solar system - especially at high ecliptic latitudes, such as highinclination, scattered objects (Brown \& Batygin 2016).

4. Because moving object trails are expected to be a significant source of astrophysical noise in the GTO/ ERS/GO observations, having more detections of these sources would provide necessary information for mitigating these aberrations.

\subsection{Science Case D: Star Formation Across Cosmic Time}

MIRI $24 \mu \mathrm{m}$ observations study star formation across cosmic time (Brown et al. 2017; Clark et al. 2018), similar to Spitzer/ MIPS or WISE/W4, but with sufficient resolution to resolve star formation regions. The $22-24 \mu \mathrm{m}$ emission in a galaxy is strongly correlated to the total star formation and can be used to accurately map local $(z<3)$ star formation rates (see, e.g., Cluver et al. 2014, 2017). Deep MIRI parallel observations will reveal where the star formation is in the galaxy populations caught by the parallel observations. A similar consideration is being made for the SPICA science cases using this wavelength regime (e.g., Bonato 2015; Gruppioni et al. 2017).

Active galactic nucleus (AGN) information is encoded in this filter as well (Jarrett et al. 2011). When MIRI is not the primary instrument for the exoplanet observations (see Figure 3), onboard data storage of MIRI default parallel imaging would not be a strong constraint. It could be the first field observations to supply star formation and AGN studies with preliminary targets. The randomized nature of the pointings allow one to constrain the numbers of rare-andbright sources.

\subsection{Science Case E: Default Parallel Time-series Observations}

Brown dwarf and exoplanet atmospheric characterization requires both high-cadence (2-200 s integrations) and long wavelength coverage $(1-10 \mu \mathrm{m})$. To discover molecular abundances in colder, smaller atmospheres, predictions show that we need JWST to be able to attain spectroscopic precisions 
on the order of $10-50 \mathrm{ppm}$ - what is referred to as "sub-50 ppm precision."

Currently, it is unknown to what precision JWST will be able to measure atmospheric features in the face of temporal, systematic noise sources; this is referred to as the "noise floor" (Greene et al. 2016; Batalha et al. 2018a). If a bright star is known in the FOV of any nonprimary instruments, while a time-series observation (TSO) is occurring on primary, and there are no immediate technical objections to conducting a second time series of a bright object $(K<15)$, then default parallels could be used to characterize the star or possibly detect a transient source. This will have a range of long-term uses from exoplanet studies to stellar characterization. The exoplanet community is attempting to attain precisions on their exoplanetary atmospheric signatures below $50 \mathrm{ppm}$ (possibly $<20$ ppm; Barstow \& Irwin 2016; Barstow et al. 2016, 2017; Greene et al. 2016; Batalha et al. 2018b; Bean et al. 2018).

With randomized FOV to observe bright objects, a critical factor in predicting the efficacy of future TSO observations is how well each stellar type (FGKM), over several wavelengths, can attain sub-50 ppm precision. Both the commissioning and ERS programs are attempting to understand this yet unknown $J W S T$ capability, but only a single target can be observed per program.

Bright enough targets for TSO observations are not typically close enough for both to fall into a second JWST instrument FOV. However, even one bright TSO target observation in the proposed default parallels program will double the number of targets observed for long temporal baselines $(>10 \mathrm{k} \mathrm{s})$. This critical information (sub-50 ppm efficacy) cannot be attained without successfully applying for a risky proposal (near saturation) in the usual GO program platform. Even one TSO target in a parallel would inform the sub-50 ppm efficacy for the entire exoplanet community.

To date, several teams predict the need for detection of atmospheric features near $10 \mathrm{ppm}$ precision (Barstow \& Irwin 2016; Barstow et al. 2016, 2017; Kreidberg 2017; Batalha et al. 2018a, and others). With the wealth of possible planetary candidates from the TESS satellite (Ricker et al. 2015), the odds of two TSO observations near each other improve dramatically (Sullivan et al. 2015). This information will provide crucial input into in the planet formation and atmospheric predictions community as well. A default parallel program with a stellar characterization/TSO component, i.e., if a bright star does fall into a second instrument's view the program would switch to TSO, would greatly inform the community to what level of precision JWST predictions can reach.

Depending on the brightness of the object $\left(m_{K} \sim 5,8,10,12\right)$, if imaging is expected to saturate, default parallels could perform grism TSO or WFSS observation instead of imaging for both NIRISS and NIRCam. This would minimize the risk of saturation and maximize the potential for stellar characterization at longer wavelengths, building up a template selection for JWST for all users.

An equally necessary benefit of default parallel TSO observations is that the greatest source of uncertainty with high-precision transiting exoplanet observations is that the stellar spectra are not as well characterized as is necessary to produce exoplanetary absolute abundance measurements. Stellar characterization through grism default parallels would benefit stellar, brown dwarf, and exoplanet observations by developing a database of stellar templates for the entire JWST program to use.

\subsection{Science Case F: Grism Observations}

As an alternative to the imaging options presented above, grism observations for the same science cases (Galactic brown dwarf population, high-redshift galaxy populations) could be considered. Deep grism stares would identify brown dwarfs (Holwerda et al. 2014; Ryan et al. 2017; Deacon 2018) as well as $z \sim 11$ galaxies (Oesch et al. 2016), of which there is a remarkable dearth (Oesch et al. 2018). The WFC3 Infrared Spectroscopic Parallel Survey (WISP) default parallel grism survey with $H S T$ /WFC3 (Atek et al. 2010) has successfully characterized the intermediate-redshift population (e.g., Atek et al. 2011, 2014; Bedregal et al. 2013; Malkan \& WISP Team 2013), predicted more use of grism spectroscopy in NIR surveys (Colbert et al. 2013), and identified some Milky Way halo objects (Masters et al. 2012). Grism observations also hold the potential to cleanly separate AGN and star formation contributions in intermediate-redshift galaxy populations (Trump et al. 2011; Bridge et al. 2016).

All four of the instruments on JWST employ slitless spectroscopic modes. In particular, NIRCam $(R \sim 1600$, $2.4<\lambda<5 \mu \mathrm{m})$ and NIRISS $(R \sim 150,0.8<\lambda<2.2 \mu \mathrm{m})$ grisms both have a wide-field mode similar to the HST WFC3 and ACS grisms. Leveraging parallel JWST observations, as has been done with $H S T$ grism surveys, will be an important undertaking to maximize scientific results. For example, doubly ionized oxygen has recently been detected at $z \sim 9$ using ALMA (Hashimoto et al. 2018), and the ability to spatially resolve [O II] out to $z \sim 12$ may revolutionize our understanding of star formation in the earliest galaxies, less than 250 million years after the Big Bang. We will also be able to probe the $[\mathrm{O}$ III] $/ \mathrm{H} \beta$ ratio out to $z \sim 9$, facilitating testing of theories about black hole seeds in the early universe and whether the existence of low-luminosity AGN, which have been found in significant numbers at $4<z<6.5$ (Giallongo et al. 2015) played a significant role in reionization.

The longer exposures without many intermezzos for default parallels would lend themselves preferentially for grism observations. 


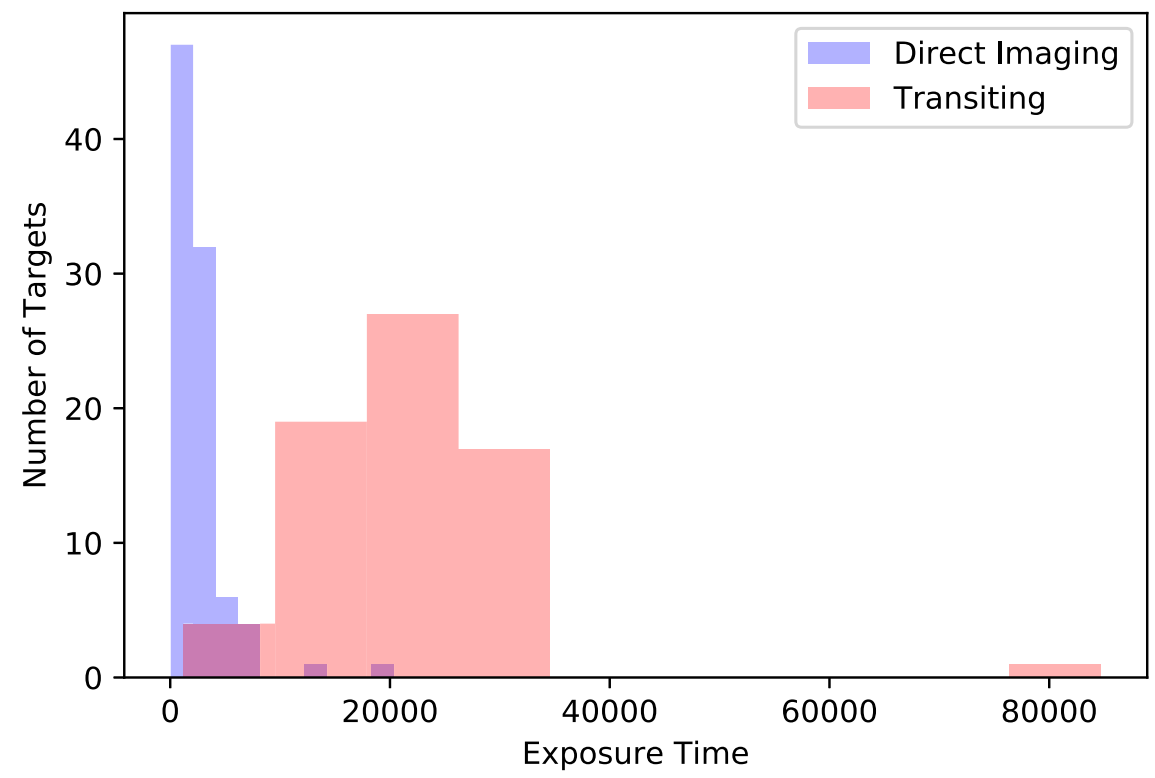

Figure 2. Exposure time histogram of the direct imaging and transiting JWST GTO programs.

(A color version of this figure is available in the online journal.)

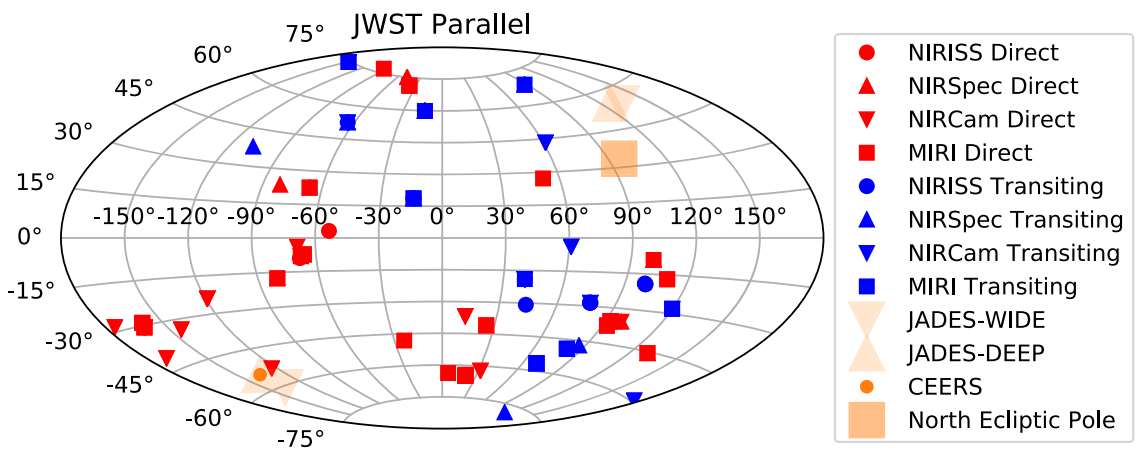

Figure 3. JWST exoplanet GTO programs projected on the sky in galactic coordinates with the primary instrument marked. (A color version of this figure is available in the online journal.)

\section{Exposure Times and Survey Area}

We consider default parallel imaging as the simplest science case, single-band observations. A TSO default parallel is likely rare, and grism observations with single orientations are more difficult to simply predict the scientific yield, but should be similar to a multiband survey.

The mean exposure time for the GTO direct imaging is $2.5 \mathrm{ks}$ and for the GTO transiting programs is $22 \mathrm{k} \mathrm{s}$. Figure 2 shows the distribution of exposure times asked for in the GTO programs. One expects GO observations to span lower integration times. The transiting programs offer the best options for a series of deep fields, possibly using multiple filters (if the transit observations include scheduled breaks, long enough to allow for filter changes).
A couple of thousand seconds of exposure time with NIRCam or NIRISS can reach depths of $m \sim 28.0$ in F200W/F277W and $M_{\mathrm{UV}} \sim-19.5$ galaxy at $9<z<13$ (see CEERS proposals, PI: Finkelstein; Finkelstein et al. 2017). The JWST default parallel observations provide the redward band for $H$-band dropout selections using $H S T$, WFIRST, or EUCLID auxiliary/follow-up data.

These default parallel observations are equivalent to the GTO's medium depth imaging surveys. Default parallels with the GTO transit observation programs could rival dedicated medium-deep fields, and over randomized FOV.

There are totals of $17 \mathrm{k}$ and $10 \mathrm{k} \mathrm{s}$ available with both NIRISS and NIRCam as the parallel instruments over 19 fields (Figures 3 and 4). Only one field looks to have too low of a 


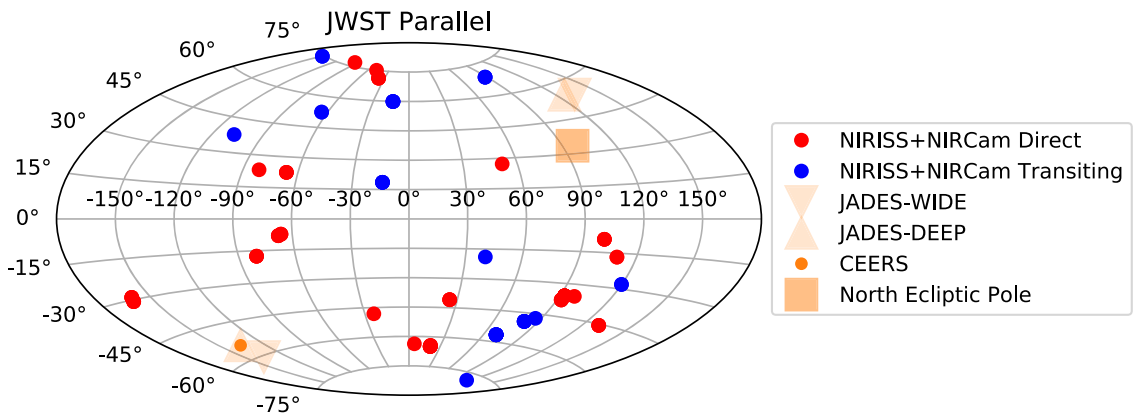

Figure 4. JWST exoplanet GTO programs projected on the sky in galactic coordinates with the availability of both NIRISS and NIRCAM as the nonprimary instrument marked.

(A color version of this figure is available in the online journal.)

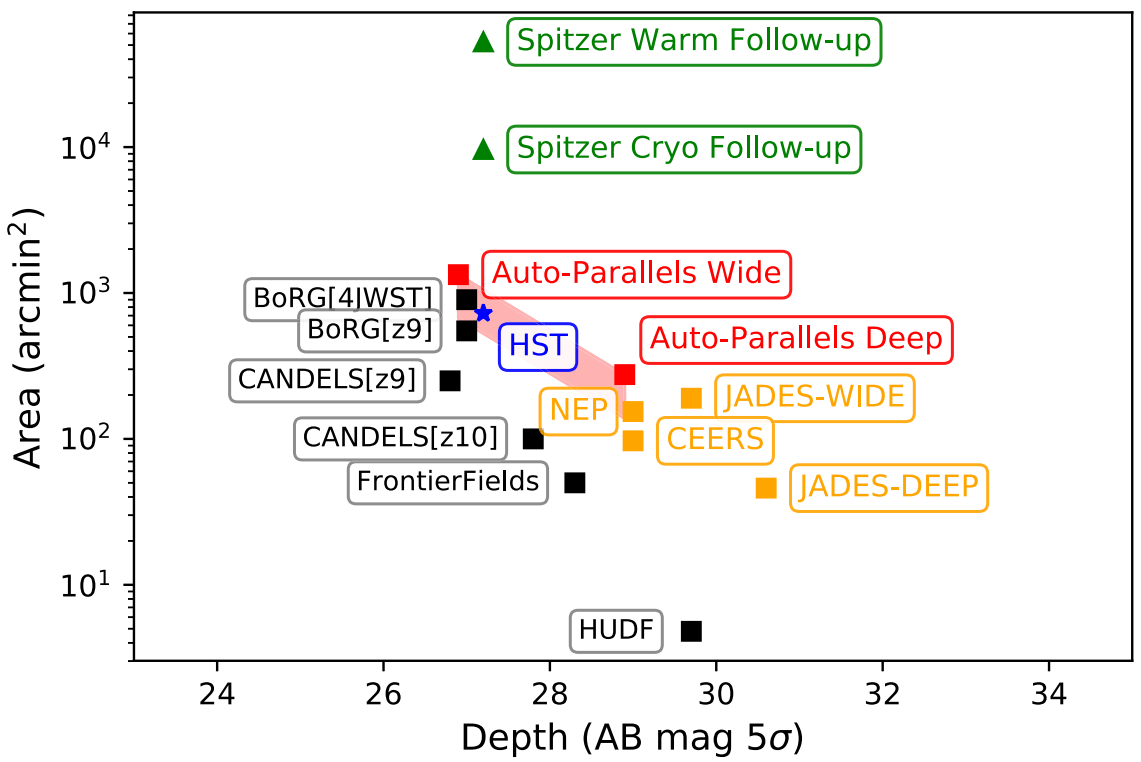

Figure 5. Approximate limiting depth and area covered by the existing HST surveys (black), the GTO CEERS survey (PI: S. Finkelstein; Finkelstein et al. 2017), JADES (PI: M. Rieke; Williams et al. 2018; Rieke et al. 2019), and NEP (PI: R. Windhorst; Jansen \& Windhorst 2018), and the possible depth and coverage of default parallels using just NIRCam and NIRISS combined pointings. The Deep and Wide scenarios are ones where the principal observation's roll angle is kept constant (deep) or varied significantly (wide). The shaded area is the range of expected depths and area covered for default parallels. These are the approximate depths and coverage for a single filter. GO observations could potentially widen the default parallel Wide option by a factor of two or more. Assuming all Spitzer Cryo and Warm mission targets are followed up with instruments other than NIRCam and NIRISS in GO programs, a shallow tier to the parallel surveys could be added.

(A color version of this figure is available in the online journal.)

Galactic latitude to be of extragalactic use, and four may suffer from stellar crowding $\left(b<15^{\circ}\right)$.

This default parallel opportunity alone would be between $275 \operatorname{arcmin}^{2}$ to almost a half a square degree survey, depending on the primary observation orientation angle constraints and the number of approved GO proposals. If the orientation angle is kept fixed, then the resulting parallel observation will be much deeper; if there is significant variation, the parallels will provide a wider survey (Figure 5).

Each of the HST deep surveys of the high-redshift universe (CANDELS, Frontier Fields, etc.) are less than $400 \operatorname{arcmin}^{2}$ each. Three GTO programs are the first deep, multiwavelength program with JWST to showcase all the instruments capabilities: the Cosmic Evolution Early Release Science (CEERS, PI: S. Finkelstein; Finkelstein et al. 2017), the JWST Advanced Deep Extragalactic Survey (JADES; Williams et al. 2018; Rieke et al. 2019), and the North Ecliptic Pole (NEP; Jansen \& Windhorst 2018) GTO deep fields.

In a single filter, default parallels would expand the discovery space significantly (Figure 5). The value of these observations will be in both comparing the known source fields to default parallels' randomized FOV, and plausibly 


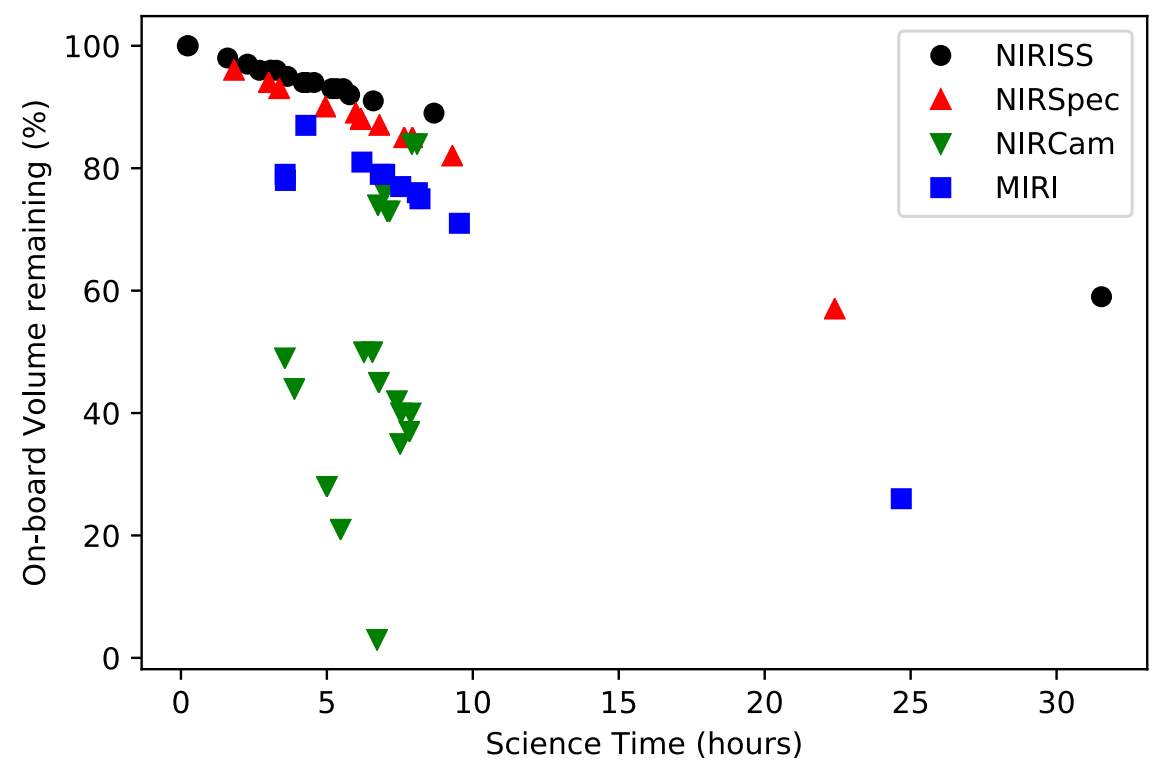

Figure 6. Remaining onboard memory for each ERS program with the primary instrument labeled. A fraction of the ERS programs use more than half the onboard memory, predominantly NIRCam programs, but two of the three very long duration ERS observations leave 50\% of the memory for potential parallel use.

(A color version of this figure is available in the online journal.)

discovering new deep targets to follow up with regular GO opportunities.

\section{Discussion}

Parallel-mode observed random pointings reduce cosmic variance more effectively than single continuous fields of a typical wedding-cake observing strategy (Trenti \& Stiavelli 2008). Hence, we argue that the opportunity for a default parallel survey will explore a unique and rich discovery space of high redshift, TSOs, solar system objects, and Galactic structure.

Random, parallel fields can counter two issues that confront high-redshift searches: cosmic variance and human bias in deep field selection. Cosmic variance remains a dominant source of uncertainty in the relatively small areas surveyed by instruments like HST and JWST (Driver \& Robotham 2010). To counter that, larger continuous areas $\left(>1^{\circ}\right)$ can be considered, but these remain observationally expensive. A randomized sampling does an equal or better job of countering cosmic variance (Trenti \& Stiavelli 2008). The current deep observations by HST, e.g., CANDELS (Grogin et al. 2011; Koekemoer et al. 2011), are all focused on those fields for which much needed ancillary data are already available. This perpetuates a bias for low Galactic cirrus regions identified decades ago. In essence, the choice for JWST fields was locked in at that time. Deep, randomly chosen fields can act as a control on deep fields dictated by legacy. Our nominal case is one that uses only the F200W or the F277W filters to perform a uniform default parallel survey. However, if there are no issues with roll angle, a series of filters can be adopted (e.g., F150W, F200W, $\mathrm{F} 277 \mathrm{~W}$, and $\mathrm{F} 356 \mathrm{~W}$ ) to combine into a photometric redshift search for high-redshift galaxies. If, for example, a transiting observation is done with intermissions, then that time can be used to switch filters in the nonprimary instruments. The principle idea for default parallels is to be a program that is executed with only a few simple rules dictating its observation strategy for ease of implementation.

There are several objections to a default parallel program that is only a single design. The program would use resources for JWST, both human and spacecraft (onboard storage and power), as well as MAST archive storage and support. Some of these are trivial but some are not and need a solid reason for their commitment. A single program would cost less but also is less flexible in its science case. It would minimize parallel use from the pool of available time for which JWST users could propose. We argue here that at present these are spacecraft hours that are not being considered since parallel observations are not allowed in concert with any nonimaging observation.

These deep exposures are also default parallel opportunities for dark exposure time. These are critical measurements of the detectors themselves, and time or parallel-mode time will need to be scheduled for separately. This need not be mutually exclusive: both a dark and a parallel observations could potentially be done during the same primary program. Only the onboard data storage limits this dual use. Figure 6 shows the science time and remaining onboard memory for the ERS programs. Most use less than $50 \%$ of the storage capability of $J W S T$, even for long exposures $(>20 \mathrm{hr}$ ) with no primary 
Publications of the Astronomical Society of the Pacific, 131:114504 (10pp), 2019 November

Holwerda et al.

Table 1

The Program ID, Main Instrument, Time, Science and Charged to Program, Storage Volume Used, Data Rate, and Percentage of Onboard Storage Still Available

\begin{tabular}{|c|c|c|c|c|c|c|c|}
\hline ProgramID & Instrument & Target & $\begin{array}{c}\text { Science } \\
\text { (hr) }\end{array}$ & $\begin{array}{c}\text { Charge } \\
\text { (hr) }\end{array}$ & $\begin{array}{l}\text { Volume } \\
\text { (GB) }\end{array}$ & $\begin{array}{c}\text { Rate } \\
\left(\mathrm{GB} \mathrm{hr}^{-1}\right)\end{array}$ & $\begin{array}{c}\text { Available } \\
(\%)\end{array}$ \\
\hline 1274 & NIRCAM & HD209458 & 6.72 & 10.07 & 56.2 & 8.4 & 3 \\
\hline 1185 & NIRCAM & HD189733 & 5.47 & 8.29 & 45.7 & 8.4 & 21 \\
\hline 1274 & NIRCAM & HD189733 & 5.0 & 8.28 & 41.8 & 8.4 & 28 \\
\hline 1274 & NIRCAM & HD149026 & 7.51 & 10.56 & 37.8 & 5.0 & 35 \\
\hline 1274 & NIRCAM & HD149026 & 7.83 & 10.56 & 36.5 & 4.7 & 37 \\
\hline 1274 & NIRCAM & HD209458 & 7.4 & 10.34 & 33.9 & 4.6 & 42 \\
\hline 1185 & NIRCAM & GJ436 (x3) & 3.89 & 5.94 & 32.5 & 8.4 & 44 \\
\hline 1274 & NIRCAM & WASP77 & 6.78 & 9.28 & 31.9 & 4.7 & 45 \\
\hline 1185 & NIRCAM & GJ436 (x3) & 3.56 & 5.94 & 29.8 & 8.4 & 49 \\
\hline 1185 & NIRCAM & WASP-80 (x2) & 6.56 & 8.91 & 29.1 & 4.4 & 50 \\
\hline 1185 & NIRCAM & WASP-80 (x2) & 6.27 & 8.91 & 29.1 & 4.6 & 50 \\
\hline 1185 & NIRCAM & HAT-P-26 & 6.75 & 9.68 & 14.9 & 2.2 & 74 \\
\hline 1177 & MIRI & НАТР19 & 8.19 & 10.58 & 14.5 & 1.8 & 75 \\
\hline 1280 & MIRI & WASP107 & 8.1 & 10.36 & 14.2 & 1.8 & 76 \\
\hline 1274 & NIRCAM & WASP77 & 6.98 & 9.28 & 13.9 & 2.0 & 76 \\
\hline 1177 & MIRI & HATP26 & 7.53 & 9.7 & 13.2 & 1.8 & 77 \\
\hline 1279 & MIRI & TRAPPIST1b (x5) & 3.58 & 4.88 & 12.5 & 3.5 & 78 \\
\hline 1177 & MIRI & TRAPPIST1b (x5) & 3.57 & 4.87 & 12.4 & 3.5 & 79 \\
\hline 1312 & MIRI & НАТР 26 & 6.98 & 9.07 & 12.3 & 1.8 & 79 \\
\hline 1177 & MIRI & WASP-80 (x2) & 6.83 & 8.89 & 12.0 & 1.8 & 79 \\
\hline 1281 & MIRI & HATP12 & 6.2 & 8.15 & 10.9 & 1.8 & 81 \\
\hline 1353 & NIRSPEC & WASP-17 (x2) & 9.3 & 11.99 & 10.3 & 1.1 & 82 \\
\hline 1185 & NIRCAM & HAT-P-19 & 7.91 & 7.8 & 9.4 & 1.2 & 84 \\
\hline 1201 & NIRSPEC & WASP107 & 5.99 & 8.3 & 6.7 & 1.1 & 89 \\
\hline 1353 & NIRISS & WASP-17 (x2) & 8.67 & 11.98 & 6.5 & 0.8 & 89 \\
\hline 1224 & NIRSPEC & GJ3053 & 4.95 & 6.98 & 5.5 & 1.1 & 90 \\
\hline 1366 & NIRISS & WASP-79 & 6.59 & 10.48 & 5.0 & 0.8 & 91 \\
\hline 1312 & NIRISS & HATP26 & 5.78 & 8.96 & 4.4 & 0.8 & 92 \\
\hline 1201 & NIRISS & K2-18 & 5.56 & 8.17 & 4.2 & 0.8 & 93 \\
\hline 1201 & NIRISS & WASP107 & 5.17 & 8.12 & 4.0 & 0.8 & 93 \\
\hline 1366 & NIRISS & WASP-18 & 5.33 & 8.66 & 3.8 & 0.7 & 93 \\
\hline 1331 & NIRSPEC & TRAPPIST-1e (x4) & 3.37 & 5.55 & 3.8 & 1.1 & 93 \\
\hline 1201 & NIRISS & HATP1 (x2) & 4.56 & 7.54 & 3.5 & 0.8 & 94 \\
\hline 1224 & NIRSPEC & WASP52 & 3.01 & 6.33 & 3.5 & 1.1 & 94 \\
\hline 1201 & NIRISS & WASP80 & 4.32 & 7.93 & 3.3 & 0.8 & 94 \\
\hline 1201 & NIRISS & LHS1140 (x2) & 4.19 & 6.47 & 3.2 & 0.8 & 94 \\
\hline 1201 & NIRISS & GJ3470 & 3.65 & 6.57 & 2.8 & 0.8 & 95 \\
\hline 1201 & NIRISS & TRAPPIST1g (x3) & 3.27 & 4.92 & 2.5 & 0.8 & 96 \\
\hline 1201 & NIRISS & HD209458 (x2) & 3.08 & 8.08 & 2.4 & 0.8 & 96 \\
\hline 1201 & NIRISS & TRAPPIST1f (x4) & 3.08 & 4.69 & 2.3 & 0.8 & 96 \\
\hline 1201 & NIRISS & WASP69 & 2.69 & 7.16 & 2.2 & 0.8 & 96 \\
\hline 1201 & NIRSPEC & TRAPPIST1d (x2) & 1.82 & 4.2 & 2.1 & 1.2 & 96 \\
\hline
\end{tabular}


Table 1

(Continued)

\begin{tabular}{lllcccc}
\hline \hline ProgramID & Instrument & Target & $\begin{array}{c}\text { Science } \\
(\mathrm{hr})\end{array}$ & $\begin{array}{c}\text { Charge } \\
(\mathrm{hr})\end{array}$ & $\begin{array}{c}\text { Volume } \\
(\mathrm{GB})\end{array}$ & $\begin{array}{c}\text { Rate } \\
(\mathrm{GB} \mathrm{hr})\end{array}$ \\
\hline 1201 & NIRISS & GJ1132 (x4) & 2.28 & 4.1 & 1.8 & 0.8 \\
1201 & NIRISS & GJ436 & 1.6 & 4.64 & 1.3 & 0.8 \\
1201 & NIRISS & HATP1 (x2) & 0.25 & 0.87 & 0.2 & 1.0 \\
1201 & NIRISS & HD209458 (x2) & 0.22 & 0.84 & 0.2 & 1.0 \\
\hline
\end{tabular}

instrument excluded. ${ }^{5}$ The remaining free space allows in principle for default parallel observations, dark frames, or both for the remaining instruments. We list the primary instrument, science and charged time, and remaining storage in Table 1 for the ERS exoplanet programs.

These observations would not be of the same quality as dedicated observations: dithering strategy-if any-will be dictated by the primary science instrument. Integration times (to limit buffer usage) could be modified to minimize data storage (i.e., DEEP8 observations). The filter choice must be fixed, because filter changes are not possible during a TSO, due to the incurred associated dither (i.e., vibrations from filter wheel motions). In the case of long-period transiting campaigns (i.e., phase-curve observations) and high-contrast imaging with multiple roll angles, then multiple filter information might be obtained in the parallel instrument as well. In the case of grism default parallel observations (if these are added to the program), roll angles will be constrained by the primary observation and no second angle will necessarily be available.

These data will not take the place of dedicated high-redshift observation campaigns that are on the docket for fields with a wealth of complementary (existing) data. Examples of similar programs with HST (ACS snapshot and the WFPC2 $B$-band pure parallel survey) build a legacy archive that often only reveal their use much later.

For example, the original ACS "schedule-gap" SNAP program (14840, PI: Bellini; Bellini 2016a, 2016b; Bellini et al. 2017) fortuitously monitored the NGC 4993 progenitor of the 2017 LIGO kilonova, which constrained the prenova mass (Cowperthwaite et al. 2017; Levan et al. 2017; Pan et al. 2017). Similarly, a dedicated WFPC2 default parallel program produced long-term value for the archive (Casertano 2002; Wadadekar et al. 2006). Such long-term value has shown to be a critical component of the HST's legacy and scientific success (e.g., Peek 2017).

The current, accepted JWST Parallel program does not permit parallel observations during transiting exoplanet observations or with multiple instruments. Individually requested parallels would require each team to decide the observation strategy per parallel field. Default parallels would

\footnotetext{
5 The NIRCam observations are the most memory intensive, but the default parallels with NIRCam need not be: these are likely only a few exposures, to rid of cosmic rays.
}

use an objective, community-designed decision tree to dictate the maximum scientific efficiency per default parallel FOV, during an exoplanet primary.

We argue here that the long exposure times necessary for exoplanet observations are an opportunity that cannot be left fallow. Either through an automated observing program-the proposed default parallels or as part of a vigorous and supported proposal process, the parallel time during exoplanet primary observations is a rich resource that needs to be exploited.

Although direct and transiting exoplanet observations are highly likely to occur throughout the lifetime of JWST, if we wait to enact this default parallel program after Cycle-1, we would lose access to parallel observations for the majority of GTO/ERS observations, the only currently existing, approved programs. This would then push back any future follow-up and characterization of plausible detections in our default parallel fields, limiting the value of each FOV. The sooner the JWST project enacts a default parallel program, the greater the legacy of these fortuitous observations will be.

We propose default parallels as a low-cost program to deliver a homogeneous and practical data set to serve a wide variety of science cases, building on the statistical strength of random sampling.

We thank Karl Gordon for the initial discussions on this subject and the suggestion to write this paper. This research made use of Astropy, a community-developed core Python package for Astronomy (Astropy Collaboration et al. 2013). This research made use of matplotlib, a Python library for publication quality graphics (Hunter 2007). PyRAF is a product of the Space Telescope Science Institute, which is operated by AURA for NASA. This research made use of SciPy (Jones et al. 2001).

\section{ORCID iDs}

B. W. Holwerda (1) https://orcid.org/0000-0002-4884-6756 Nelly Mouawad (ib https://orcid.org/0000-0003-1609-5625 Joanna S. Bridge (ㄱ) https://orcid.org/0000-0002-8584-1903

\section{References}

Astropy Collaboration, Robitaille, T. P., Tollerud, E. J., et al. 2013, A\&A, 558, A33

Atek, H., Kneib, J.-P., Pacifici, C., et al. 2014, ApJ, 789, 96

Atek, H., Malkan, M., McCarthy, P., et al. 2010, ApJ, 723, 104 
Atek, H., Siana, B., Scarlata, C., et al. 2011, ApJ, 743, 121

Barstow, J. K., Aigrain, S., Irwin, P. G. J., \& Sing, D. K. 2017, ApJ, 834, 50

Barstow, J. K., \& Irwin, P. G. J. 2016, MNRAS, 461, L92

Barstow, J. K., Irwin, P. G. J., Kendrew, S., \& Aigrain, S. 2016, Proc. SPIE, 9904, 99043P

Batalha, N. E., Lewis, N. K., Line, M. R., Valenti, J., \& Stevenson, K. 2018a, ApJL, 856, L34

Batalha, N. E., Smith, A. J. R. W., Lewis, N. K., et al. 2018b, AJ, 156, 158 Batygin, K., \& Brown, M. E. 2016, ApJL, 833, L3

Bean, J. L., Stevenson, K. B., Batalha, N. M., et al. 2018, PASP, 130, 114402

Bedregal, A. G., Scarlata, C., Henry, A. L., et al. 2013, ApJ, 778, 126

Bellini, A. 2016a, Extended F814W Schedule Gap Pilot, HST Proposal, 15364B

Bellini, A. 2016b, Schedule Gap Pilot, HST Proposal, 14840B

Bellini, A., Grogin, N. A., Hathi, N., \& Brown, T. A. 2017, Instrument Science Report

Bernard, S. R., Carrasco, D., Trenti, M., et al. 2016, ApJ, 827, 76

Bonato, M. 2015, PhD thesis, University of Padua

Bradley, L. D., Trenti, M., Oesch, P. A., et al. 2012, ApJ, 760, 108

Bridge, J. S., Zeimann, G. R., Trump, J. R., et al. 2016, ApJ, 826, 172

Brown, M. E., \& Batygin, K. 2016, ApJL, 824, L23

Brown, M. J. I., Moustakas, J., Kennicutt, R. C., et al. 2017, ApJ, 847, 136

Calvi, V., Trenti, M., Stiavelli, M., et al. 2016, ApJ, 817, 120

Cameron, A. J., Trenti, M., Livermore, R. C., \& van der Velden, C. 2019, MNRAS, 483, 1922

Casertano, S. 2002, The WFPC2 Archival Parallels, HST Proposal, 9540C

Clark, C. J. R., Verstocken, S., Bianchi, S., et al. 2018, A\&A, 609, A37

Cluver, M. E., Jarrett, T. H., Dale, D. A., et al. 2017, ApJ, 850, 68

Cluver, M. E., Jarrett, T. H., Hopkins, A. M., et al. 2014, ApJ, 782, 90

Colbert, J. W., Teplitz, H., Atek, H., et al. 2013, ApJ, 779, 34

Cowperthwaite, P. S., Berger, E., Villar, V. A., et al. 2017, ApJL, 848, L17

Deacon, N. R. 2018, MNRAS, 481, 447

Driver, S. P., \& Robotham, A. S. G. 2010, MNRAS, 407, 2131

Fernández, Y. R., Kelley, M. S., Lamy, P. L., et al. 2013, Icar, 226, 1138

Finkelstein, S., Dickinson, M., Ferguson, H., et al. 2017, The Cosmic Evolution Early Release Science (CEERS) Survey, JWST Proposal ID 1345. Cycle 0 Early Release Scienc

Giallongo, E., Grazian, A., Fiore, F., et al. 2015, A\&A, 578, A83

Greene, T. P., Line, M. R., Montero, C., et al. 2016, ApJ, 817, 17

Grogin, N. A., Kocevski, D. D., Faber, S. M., et al. 2011, ApJS, 197, 35

Gruppioni, C., Ciesla, L., Hatziminaoglou, E., et al. 2017, PASA, 34, e055

Hashimoto, T., Laporte, N., Mawatari, K., et al. 2018, Natur, 557, 392

Holwerda, B. W., Bridge, J. S., Ryan, R., et al. 2018, A\&A, 620, A132

Holwerda, B. W., Trenti, M., Clarkson, W., et al. 2014, ApJ, 788, 77

Hunter, J. D. 2007, CSE, 9, 90

Jansen, R. A., \& Windhorst, R. A. 2018, PASP, 130, 124001

Jarrett, T. H., Cohen, M., Masci, F., et al. 2011, ApJ, 735, 112
Jones, E., Oliphant, T., Peterson, P., et al. 2001, SciPy: Open source scientific tools for Python

Kelley, M. S., Fernández, Y. R., Licandro, J., et al. 2013, Icar, 225, 475

Kiss, C., Pál, A., Müller, T. G., \& Ábrahám, P. 2008, A\&A, 478, 605

Koekemoer, A. M., Faber, S. M., Ferguson, H. C., et al. 2011, ApJS, 197, 36

Kreidberg, L. 2017, Exoplanet Atmosphere Measurements from Transmission Spectroscopy and Other Planet Star Combined Light Observations, 100

Levan, A. J., Lyman, J. D., Tanvir, N. R., et al. 2017, ApJL, 848, L28

Livermore, R. C., Trenti, M., Bradley, L. D., et al. 2018, ApJL, 861, L17

Malkan, M., \& WISP Team 2013, in ASP Conf. Ser. 477, Galaxy Mergers in an Evolving Universe, ed. W.-H. Sun et al. (San Francisco, CA: ASP), 255

Masters, D., McCarthy, P., Burgasser, A. J., et al. 2012, ApJL, 752, L14

Meadows, V. S., Bhattacharya, B., Reach, W. T., et al. 2004, ApJS, 154, 469

Morishita, T., Trenti, M., Stiavelli, M., et al. 2018, ApJ, 867, 150

Oesch, P. A., Bouwens, R. J., Illingworth, G. D., Labbé, I., \& Stefanon, M. 2018, ApJ, 855, 105

Oesch, P. A., Brammer, G., van Dokkum, P. G., et al. 2016, ApJ, 819, 129

Pan, Y.-C., Kilpatrick, C. D., Simon, J. D., et al. 2017, ApJL, 848, L30

Peek, K. 2017, SciAm

Petit, J. M., Kavelaars, J. J., Gladman, B. J., et al. 2008, Sci, 322, 432

Petit, J.-M., Kavelaars, J. J., Gladman, B. J., et al. 2011, AJ, 142, 131

Pirzkal, N., Burgasser, A. J., Malhotra, S., et al. 2009, ApJ, 695, 1591

Pirzkal, N., Sahu, K. C., Burgasser, A., et al. 2005, ApJ, 622, 319

Ricker, G. R., Winn, J. N., Vanderspek, R., et al. 2015, JATIS, 1, 014003

Rieke, M. Arribas, S., Bunker, A., et al. 2019, BAAS, 51, 45

Ryan, E. L., Mizuno, D. R., Shenoy, S. S., et al. 2015, A\&A, 578, A42

Ryan, R. E., Thorman, P. A., Yan, H., et al. 2011, ApJ, 739, 83

Ryan, R. E., Jr., Hathi, N. P., Cohen, S. H., \& Windhorst, R. A. 2005, ApJL, 631, L159

Ryan, R. E., Jr., Thorman, P. A., Schmidt, S. J., et al. 2017, ApJ, 847, 53

Stansberry, J. A., Van Cleve, J., Reach, W. T., et al. 2004, ApJS, 154, 463

Steidel, C. C., Giavalisco, M., Dickinson, M., \& Adelberger, K. L. 1996, AJ, 112,352

Sullivan, P. W., Winn, J. N., Berta-Thompson, Z. K., et al. 2015, ApJ, 809, 77

Trenti, M. 2014, Bright Galaxies at Hubble's Detection Frontier: The redshift $\mathrm{z} \sim 9$-10 BoRG pure-parallel survey, HST Proposal

Trenti, M., Bradley, L. D., Stiavelli, M., et al. 2011, ApJL, 727, L39

Trenti, M., Bradley, L. D., Stiavelli, M., et al. 2012, ApJ, 746, 55

Trenti, M., \& Stiavelli, M. 2008, ApJ, 676, 767

Trilling, D. E., Mommert, M., Hora, J., et al. 2016, AJ, 152, 172

Trilling, D. E., Valdes, F., Allen, L., et al. 2017, AJ, 154, 170

Trump, J. R., Weiner, B. J., Scarlata, C., et al. 2011, ApJ, 743, 144

van Vledder, I., van der Vlugt, D., Holwerda, B. W., et al. 2016, MNRAS, 458,425

Wadadekar, Y., Casertano, S., Hook, R., et al. 2006, PASP, 118, 450

Williams, C. C., Curtis-Lake, E., Hainline, K. N., et al. 2018, ApJS, 236, 33

Wright, E. L., Eisenhardt, P. R. M., Mainzer, A. K., et al. 2010, AJ, 140, 1868 